\title{
Traduire l'étrusque : problématiques antiques et modernes
}

\author{
Yves Liébert \\ Université de Limoges
}

\section{Introduction}

L'héritage étrusque de Rome est vaste et bien connu : les sources littéraires gréco-romaines, tout comme l'archéologie, nous en fournissent de nombreux témoignages. La période des rois étrusques de Rome, que la tradition situe entre 616 et 509 avant J.-C. avec Tarquin l'Ancien, Servius Tullius et Tarquin le Superbe, fut incontestablement une grande période d'aménagements et de développement de l'Vrbs. Cette domination étrusque sur la future métropole laissa, bien après le VI ${ }^{\mathrm{ème}}$ siècle avant J.-C., des traces profondes dans le monde romain, que ce soit dans le domaine institutionnel ou religieux, dans la vie quotidienne et les mentalités ou, plus largement, dans la géographie italienne.

Ceux qui furent quelques temps les maîtres de Rome et d'une grande partie de la Péninsule se distinguaient cependant par une langue fort différente du latin et des langues indo-européennes en général. Le problème de la connaissance de la langue étrusque à Rome et de sa traduction est donc un point crucial pour juger des interactions entre la culture étrusque et la culture romaine.

Nous verrons dans un premier temps combien il est difficile de rassembler une documentation suffisante pour poser cette problématique dans l'Antiquité et analyser avec pertinence la situation. Nous développerons dans un second temps les conséquences que cette difficulté initiale a induites sur la traduction de l'étrusque à l'époque moderne.

\section{La langue étrusque dans l'antiquité}

L'un des premiers problèmes qui se posent à l'étruscologue est que la langue des anciens Toscans n'est attestée que par l'épigraphie. Cela ne veut pas dire qu'une littérature en langue étrusque n'ait pas existé, mais nous 
n'avons guère que les traces d'une littérature religieuse et aucun texte de littérature profane ne nous est parvenu : nous sommes donc obligés de nous contenter de témoignages indirects et délicats à interpréter.

\section{La documentation}

Rappelons tout d'abord que l'état de notre documentation sur la langue étrusque est plutôt paradoxal car, pour la période qui s'étend de 700 à 10 avant J.-C., l'étruscologue dispose d'un corpus d'environ 11000 inscriptions, ce qui est bien supérieur au nombre de documents épigraphiques latins que nous possédons pour la même période. Encore faut-il préciser que la plupart de ces inscriptions en langue étrusque sont de nature funéraire, courtes et, très souvent, suivent des formulaires répétitifs. Les plus longs documents sont conservés sur des supports faits de matériaux variés - terre cuite, pierre, métal et tissu - et sont de natures diverses : les quatre textes les plus longs se trouvent sur un cippe provenant de Pérouse, une table de Cortone, une tuile de Capoue conservée au musée de Berlin et une momie égyptienne du musée de Zagreb.

Le cippe de Pérouse et la table de Cortone sont manifestement des documents de nature juridique. Le premier est une grande pierre de bornage (tular en étrusque) qui comporte le texte d'un jugement rendu entre deux familles de propriétaires étrusques, les Velthina et les Afuna, et qui concerne la limitation de leurs propriétés respectives. L'arbitrage semble avoir été rendu par un personnage, étranger à ces deux familles, qui porte le nom de Tanna Larezu et est qualifié de teurat, terme qui apparaît aussi sur des scènes sportives de la peinture tarquinienne pour désigner un arbitre ${ }^{1}$. Ce document de 126 mots est assez tardif et remonte au III ${ }^{\text {ème }}$ ou au II ${ }^{\text {ème }}$ siècle avant J.-C.

La table de Cortone est aussi un document de nature juridique pour ce qu'on en comprend. Cette plaque de bronze de $45 \mathrm{~cm}$ sur 30 , dont nous n'avons conservé que 7 fragments sur 8 , présente 32 lignes de texte étrusque sur son recto et 8 lignes au verso : c'est donc le troisième texte étrusque conservé par sa longueur. Les éditeurs de cette trouvaille récente, qui n'a été publiée qu'en 2000, L. Agostiniani et F. Nicosia ${ }^{2}$, datent ce texte de 200 avant J.-C. et le considèrent comme un contrat portant sur la location ou la vente de terrains situés sur le territoire de Trasimène dont le nom semble apparaître sur la face B de l'inscription (tarsminas). Ce document est constitué pour plus de sa moitié de noms propres (106 très précisément, sur les 206 mots de ce document) répartis en quatre listes dans lesquelles on s'est ingénié à repérer vendeurs, acheteurs ou magistrats garants de la transaction. Notons que certains vendeurs appartiendraient à une famille bien connue de Cortone, les 
Cusu, et que, outre les noms des acquéreurs (ou locataires) apparaissent aussi les mentions de magistrats, dont un zilat mexl rasnal, titre que l'on a longtemps associé à la fonction de magistrat suprême de la «fédération » étrusque, mais qui pourrait être, plus simplement, le magistrat supérieur de la seule cité de Cortone. ${ }^{3}$ On retrouve aussi des formules comme zilci lar $\theta a l$ cusus titinal larisalc salinis aulesla dans laquelle on peut repérer le terme zilci : "Sous la magistrature de Larth Culsu fils de Titinei (matronyme) et de Laris Salinis fils de Aule », formule de datation par magistrats éponymes dont on connait bien l'équivalent dans le monde romain (M. Tullio C. Antonio consulibus pour 63 avant J.-C. par exemple).

Les deux autres textes sont vraisemblablement, quant à eux, de nature religieuse.

La tuile de Capoue est un document beaucoup plus ancien puisqu'elle remonte au $\mathrm{V}^{\text {ème }}$ siècle avant J.-C. (vers 470 plus précisément). Même si le texte n'est pas parfaitement compréhensible pour nous, on constate cependant qu'il s'agit d'un calendrier religieux dont les dix sections encore visibles (soit 300 mots) comportent tout un rituel avec des prescriptions et le détail d'offrandes à faire à telle date à telle divinité. K. Olzscha ${ }^{4}$ et, plus récemment, H. Rix ${ }^{5}$ et M. Cristofani ${ }^{6}$, ont pu démontrer la nature de ces formules de datation et les analyser en détail : ainsi le texte de la tuile nous apprend-il qu'il faut faire une offrande de trois tartiria et de trois cleva au dieu Lethams ${ }^{7}$ : cette divinité est connue dans le panthéon étrusque; en revanche, il faut avouer notre ignorance sur ce que sont les tartiria et les cleva!

C'est aussi un calendrier religieux qu'offre le texte de la momie de Zagreb, qui est le plus long rédigé en langue étrusque qui nous soit parvenu avec ses quelque 1200 mots. Les hasards de l'histoire font que ce document nous a été transmis par l'intermédiaire de la momie d'une femme morte et embaumée à l'époque tardo-ptolémaïque ou à l'époque romaine. Celle-ci fut achetée au XIX ${ }^{\text {ème }}$ siècle par un Croate établi à Vienne, Michel de Baric, qui faisait, comme tous les gens un peu fortunés et aventureux de l'époque, un tour des grands sites antiques de la Méditerranée occidentale et orientale dans la tradition du «Grand Tour» des aristocrates du XVIII ${ }^{\text {ème }}$ siècle. Il vint en Égypte en 1848-1849, y acheta ce qui n'était qu'un souvenir des plus représentatifs de son séjour sur la terre des anciens pharaons et retourna en Autriche. À sa mort, en 1859, son frère Elias hérita de la momie et en fit don au musée de la ville où il vivait, Zagreb. C'est ainsi qu'une momie achetée en Égypte arriva dans un musée croate dont le conservateur se rendit vite 
compte que les bandelettes portaient un texte tracé à l'encre. De fait, la momie était enveloppée dans un tissu de lin et, en 1891, le Viennois J. Krall, plus perspicace que ceux qui s'y étaient intéressés avant lui, comprit qu'il s'agissait d'un document rédigé en étrusque. Il publia aussitôt une première lecture du texte qui avait été, de fait, consigné sur un support périssable dont les auteurs anciens et l'archéologie nous apprennent que les Étrusques se servaient pour recopier des textes sacrés: une toile de lin. Les libri lintei des anciens Toscans nous sont effectivement connus par le témoignage de Tite-Live ${ }^{8}$ ou encore par l'archéologie. F. Roncalli a reconnu de tels documents représentés sur certains sarcophages étrusques attribués à des personnages religieux : ils ressemblent à un drap replié plusieurs fois sur lui-même, la face portant le texte étant située à l'intérieur afin de le protéger ${ }^{9}$. De toute évidence, ce livre de lin avait été introduit en Égypte par un Étrusque ou un individu étruscophone qui en connaissait la signification religieuse : l'on a songé naturellement à l'un de ces haruspices, spécialistes de l'Etrusca disciplina, qui, intégrés dans le monde romain, suivaient en particulier les armées aux quatre coins du monde et emportaient avec eux des copies de leurs livres sacrés $^{10}$. La seule certitude que nous ayons, c'est que ce livre, qui fut finalement déchiré en bandelettes pour envelopper un corps, date du début du premier siècle avant J.-C., selon les analyses paléographiques. Qu'on utilisât encore ce support, dont l'usage était répandu en Italie avant l'introduction du papyrus ou du parchemin, renvoie manifestement à la tradition religieuse étrusque.

Pour le contenu, il nous reste aujourd'hui la trace de douze colonnes, dont la première est presque totalement perdue, qui livrent un calendrier rituel très précis ordonné selon des dates qui ouvrent chaque section. Il s'agit là encore d'une liste de prescriptions religieuses indiquant les cérémonies à accomplir et les dieux pour lesquels elles se font.

Même si, malgré ces textes longs, notre connaissance de la langue étrusque reste très lacunaire, on ne peut douter que les anciens Toscans conservaient par écrit à travers les siècles de nombreuses traditions et connaissances, en particulier dans le domaine religieux. Que pouvons-nous dire de la littérature étrusque, hormis les textes sacrés ?

\section{Textes étrusques}

L'absence de littérature profane qui nous soit parvenue peut s'expliquer autrement que par les seuls hasards de l'histoire et de la transmission des textes : Jacques Heurgon ${ }^{11}$ a fort bien mis en avant que les élites étrusques, fortement hellénisées, pouvaient s'être essayées, comme le 
firent aussi bien les Romains, à une littérature en langue grecque en ne conservant leur propre langue que pour les écrits religieux.

Nous avons cependant quelques indices qui permettent de dépasser cette perspective.

D’une façon assez générale, Posidonius d'Apamée (135-50 avant J.C.), dans une vaste notice de ses Histoires conservée par Diodore de Sicile et portant sur les Étrusques, mentionne les lettres étrusques en les distinguant de la théologie :

Gr£mmata dè $\mathrm{ka}^{\wedge}$ fusiolog...an ka^ qeolog...an ${ }^{\mathrm{TM}}$ xepÒnhsan ${ }^{\mathrm{TM}} \mathrm{p}^{\wedge}$ plšon, $\mathrm{ka}^{\wedge} \mathrm{t}_{1}^{1}$ per $\mathrm{t}^{3} / 4 \mathrm{n}$ keraunoskop...an m£lista p£ntwn Łnqrèpwn ${ }^{\mathrm{TM}}$ xeirg£santo :

«Les lettres, les sciences de la nature et la théologie, ils les firent progresser et ils développèrent plus que tous les autres hommes ce qui concerne l'observation des foudres. ${ }^{12}$

Mais l'érudit augustéen Varron cite plus précisément un certain Volnius qui aurait «écrit des tragédies étrusques » ${ }^{13}$. Cet auteur au gentilice étrusque répandu (Velna ou Velina), qui a dû vivre à l'époque des Gracques, était intervenu dans la discussion étymologique sur les trois tribus primitives romaines faisant venir tribus de tris et c'est à cette occasion que Varron nous le présente aussi comme un auteur dramatique :

Ager Romanus primum diuisus in partis tris, a quo tribus appellata Titiensium, Ramnium, Lucerum. Nominatae, ut ait Ennius, Titienses ab Tatio, Ramnenses ab Romulo, Luceres, ut Iunius, ab Lucumone; sed omnia haec vocabula tusca, ut Volnius, qui tragoedias tuscas scripsit, dicebat.

«L'ager Romanus fut partagé à l'origine en trois régions, d'où le nom de tribus des Titienses, des Ramnes et des Luceres. Désignation due, selon Ennius, pour les Titienses à Tatius, pour les Ramnenses à Romulus, et pour les Luceres, selon Junius, à Lucumon ; mais tous ces mots-là, Volnius, qui a écrit des tragédies en étrusque, les déclarait étrusques. ${ }^{14}$

Ce n'est pas le lieu de discuter ici du caractère étrusque des noms des trois tribus romuléennes, mais cette mention permet de poser le problème du sens même de l'expression de tragoediae tuscae utilisée par Varron.

J. Heurgon pensait à des pièces rédigées en étrusque qui eussent été le fait d'un personnage «très fier de ses origines nationales » : ces tragédies étrusques auraient été, selon lui, des « œuvres d'érudition archaïsante qui $\varepsilon r$ 
furent peut-être jouées sur les derniers tréteaux de Chiusi ou de Volterra et y ranimèrent in extremis un feu en voie de s'éteindre ${ }^{15} »$. Cette interprétation favorable à l'existence de pièces en langue étrusque reste toutefois bien hypothétique. Certes, J. Heurgon avançait un autre élément en faveur de l'existence de ces tragédies étrusques à partir d'un nom qui apparaît dans le Miles gloriosus de Plaute: ce savant repérait dans l'étrange Clutumistaridysarchides un composé étrusco-grec dont le premier élément est la forme étrusque de Clytemnestre (clutumsta) ${ }^{16}$, nom que Plaute aurait pu tirer de quelque pièce étrusque ; mais là encore, nous restons dans le domaine de l'hypothèse.

En fait, l'on peut tout aussi bien comprendre l'expression tragoediae Tuscae de Varron comme «tragédies à sujet d'inspiration étrusque », plutôt que comme «tragédies rédigées en étrusque», ce qui n'implique pas nécessairement, dans ce cas, que les pièces soient rédigées dans cette langue : c'est la lecture que fait F.-H. Massa-Pairault ${ }^{17}$ de l'expression du Réatin. Et même si les bas-reliefs des urnes hellénistiques de Pérouse et de Volterra présentent bien des décors qui s'apparentent à ceux du théâtre, même si la découverte d'un théâtre de pierre équipé de gradins pour accueillir des spectateurs à Castelsecco, près d'Arezzo ${ }^{18}$, ne laisse aucun doute sur l'existence de représentations théâtrales, la date tardive de ces témoignages archéologiques (III ${ }^{\mathrm{ème}}$ au $\mathrm{I}^{\mathrm{er}}$ siècle avant J.-C.) ne peut garantir l'existence d'un théâtre étrusque antérieur au théâtre romain et, de surcroît, en langue étrusque.

Ajoutons enfin que, comme le fait remarquer D. Briquel ${ }^{19}$, le fait que Volnius se soit intéressé à un sujet aussi typiquement romain que les tribus romuléennes laisse penser qu'il ne peut s'agir d'un auteur ancien : aurait-il voulu, à une époque où l'Étrurie était bien intégrée au monde romain, sauver ainsi la langue de ses ancêtres ou même conférer une dimension étrusque aux origines de Rome ou, au contraire, n'était-il pas très fortement influencé par Rome au point qu'il soit douteux qu'il s'ingéniât à composer des pièces en étrusque?

Le problème d'une littérature profane en langue étrusque se pose d'ailleurs dans les mêmes termes avec l'historiographie : là encore, c'est Varron qui mentionne des Tuscae historiae qui recouvrent peut-être une historiographie étrusque perdue ${ }^{20}$. Le témoignage le plus net en faveur de l'existence de cette littérature historique est celui de l'empereur Claude. Le rapport de Claude aux anciens Toscans ne se limite effectivement pas au regain d'intérêt pour les Étrusques qui s'est manifesté à la fin du I ${ }^{\text {er }}$ siècle avant J.-C. à travers des personnalités et des œuvres comme celles de Mécène, 
Virgile, Verrius Flaccus ou Denys d'Halicarnasse. D. Briquel le qualifie d' «empereur-étruscologue » ${ }^{21}$. En fait, seul Suétone ${ }^{22}$ nous dit que Claude aurait écrit en grec vingt livres sur les Étrusques, des Tyrrhènika dont il aurait fait faire des lectures publiques annuelles dans le Musée d'Alexandrie en alternance avec les huit livres d'une histoire de Carthage dont il était aussi l'auteur. Mais son mariage avec Plautia Urgulanilla, d'ascendance toscane, lui permettait de disposer dans le cadre familial d'une information de première main sur les Étrusques comme le rappelait J. Heurgon ${ }^{23}$. Et c'est en s'appuyant sur des auctores Tusci qu'il utilise, dans son discours au sénat de 48, gravé sur la table de Lyon $^{24}$, la version étrusque de la geste de Caeles Vibenna et de Mastarna, autrement dit Servius Tullius, corroborée par les peintures de la tombe François de Vulci ${ }^{25}$. Il est intéressant de voir que Claude s'appuie sur une version différente de celle que l'annalistique romaine avait rendu canonique pour défendre sa politique du moment et allie à sa pratique de dirigeant l'érudition qui avait déjà marqué sa jeunesse ; il veut alors justifier la concession du ius honorum à des Gaulois en s'appuyant sur les antiques précédents de Rome même qui avait vu des étrangers tels Numa, Tarquin et Servius la diriger. Le discours tenu par Claude au sénat et rapporté par Tacite insiste, lui, sur la nécessité de ne pas « laisser perdre par négligence la science la plus ancienne de l'Italie ${ }^{26}$. Cela se traduira concrètement par la réorganisation de l'ordre des soixante haruspices en 47. Mais ce qui nous importe ici, c est que Claude ait eu accès à des sources étrusques, peut-être à certaines de ces archives gentilices étrusques dont les elogia Tarquiniensia, inscriptions latines du ${ }^{\mathrm{er}}$ siècle avant J.-C., sont de récents avatars ${ }^{27}$.

Toutefois, s'il est difficile de retrouver les traces des lettres étrusques dans le domaine profane, la littérature religieuse des anciens Toscans, elle, nous est mieux connue.

\section{Apprentissage et traduction de l'étrusque à Rome}

C'est en effet aux livres sacrés de l'Etrusca disciplina, dont le livre de lin de Zagreb nous fournit un exemple concret, que fait avant tout penser la mention de lettres étrusques. Religion révélée, avec ses prophètes tels Tagès ou la nymphe Vegoia, la religion étrusque était une religion du Livre, fait fondamentalement étranger aux auteurs grecs et romains qui n'ont pas manqué de mettre en avant cette particularité ; la religion romaine avait intégré la science religieuse contenue dans ces libri haruspicini, libri fulgurales ou libri rituales que mentionnent les sources gréco-latines et Tite¿० 
Live n'hésite d'ailleurs pas à présenter les anciens Toscans comme les plus religieux des hommes ${ }^{28}$.

De fait, il n'est pas douteux que les élites romaines ont pu maitriser depuis longtemps la langue étrusque.

D'abord, parce que nous savons, preuves linguistiques à l'appui, que l'alphabet latin est tiré de l'alphabet grec par l'intermédiaire des Étrusques et on devine le rôle d'élites bilingues dans ce domaine. En effet, la phonologie étrusque ne comporte pas de sonores : ainsi, l'occlusive vélaire sonore $[\mathrm{g}]$ transcrite par la troisième lettre de l'alphabet grec, le $\gamma$, se voit attribuer en étrusque une autre valeur, celle de l'occlusive vélaire sourde [k]. Or l'alphabet latin, et par conséquent de nos jours l'alphabet français, comporte en troisième place un $\mathrm{C}$ qui n'est plus une sonore $[\mathrm{g}]$ comme l'était le gamma grec, mais une sourde [k] qui suppose bien le passage par l'étrusque, puisque les Latins, dont la langue possède le phonème [g], l'ont noté non pas avec le gamma qui avait changé de valeur, mais avec un nouveau signe, le $\mathrm{G}$, c'est-à-dire un $\mathrm{C}$ distingué de la sourde par l'ajout d'une petite barre ${ }^{29}$.

Ensuite, les sources littéraires nous apprennent que les fils de bonne famille de Rome se formaient aux lettres étrusques. Tite-Live, dans un passage célèbre du livre IX de son Histoire romaine, nous explique que les jeunes Romains allaient se former aux lettres étrusques en Étrurie, comme ils le feront plus tard aux lettres grecques en Grèce :

Habeo auctores uulgo tum Romanos pueros, sicut nunc Graecis, ita Etruscis litteris erudiri solitos.

«J'ai des sources qui prouvent que c'était l'habitude en ce temps-là d'instruire les jeunes Romains dans les lettres étrusques comme on le fait aujourd'hui dans les lettres grecques. ${ }^{30}$

J. Heurgon a bien montré qu'il s'agissait non seulement d'apprendre la langue, mais aussi la littérature étrusque, quelle que fût sa nature ${ }^{31}$. Il est intéressant de rappeler le contexte de cette citation de Tite-Live. Au moment où Rome se lance à la conquête de l'Étrurie, un membre de la gens Fabia parvient à effectuer une reconnaissance en pays étrusque, opération qui aboutira au franchissement de la forêt Ciminienne et à la réussite de l'expédition militaire de 310 , grâce à sa connaissance de la langue étrusque. Tite-Live nous précise bien que, "élevé à Caeré chez des hôtes, [Fabius] avait reçu l'enseignement étrusque, et connaissait bien la langue étrusque », tout comme, d'ailleurs, l'esclave qui fut son seul compagnon dans cette mission de reconnaissance et qui, «élevé avec lui, ... n'ignorait pas non plus la langue étrusque ». Retenons ici le rôle de capitale intellectuelle que pouvait 
jouer alors une riche cité étrusque comme Caeré, qui était ouverte vers le monde grec, mais échangeait aussi et entretenait des liens avec Rome ${ }^{32}$. L'épigraphie va d'ailleurs dans le sens de liens privilégiés de certaines gentes romaines, et en particulier de la gens Fabia, avec certaines cités étrusques et notamment Caeré : elle nous révèle la présence dans cette cité d'un Kalatur Phapena, qui est un Calator Fabius, dès le VII ${ }^{\text {ème }}$ siècle ${ }^{33}$.

Cela fait donc longtemps que les élites romaines sont, pour une part, imprégnées de culture étrusque quand, à l'époque de Cicéron, les Romains se soucient de traduire en latin les œuvres rédigées dans une langue dont le nombre de locuteurs ne cesse de décroître.

Ces traducteurs semblent être le plus souvent d'origine toscane, comme c'est le cas pour Aulus Caecina, issu d'une grande famille de Volterra et ami de Cicéron, qui connaissait des textes religieux originaux et les traduisit en latin dans un De Etrusca disciplina, utilisé par Cicéron dans son De diuinatione et, ensuite, par Sénèque et Pline l'Ancien. C'est aussi le cas de Lucius Tarquitius Priscus, auteur d'un De rebus diuinis - les Tarquitiani libri qui contribuèrent à diffuser l'Etrusca disciplina à Rome - et qui était vraisemblablement un ami de Varron ${ }^{34}$. Ces traductions latines d'un Caecina, d'un Tarquitius ou encore d'un Julius Aquila, sont hélas elles aussi perdues et nous n'en connaissons que les fragments cités par quelques auteurs latins. De même Nigidius Figulus avait-il lui aussi traduit un document religieux, un liber fulguralis, c'est-à-dire un calendrier brontoscopique indiquant la signification des coups de tonnerre pour tous les jours de l'année. Ce texte nous est connu par la traduction faite en grec byzantin par Jean le Lydien au milieu du VI ${ }^{\text {ème }}$ siècle après J.-C. ${ }^{35}$.

Ces entreprises de traduction de textes étrusques, qui semblent se multiplier entre le $\mathrm{I}^{\text {er }}$ siècle avant et le $\mathrm{I}^{\text {er }}$ siècle après J.-C., peuvent ressembler à une tentative de conservation d'un patrimoine cher aux Romains, mais voué à la disparition au moment même où l'étrusque devient une langue morte. Toutefois, l'engouement pour la science religieuse étrusque, si ce n'est pour la culture étrusque en général, que traduit ce mouvement, s'accompagne, heureusement pour nous, d'un souci régulier des auteurs anciens de rappeler l'héritage lexical étrusque de Rome.

Nous ne pouvons citer ici tous les termes auxquels les Romains attribuaient une origine étrusque: les emprunts étrusques en latin, notamment, ont été très bien étudiés par $\mathrm{G}$. Breyer ${ }^{36}$, et nous rappellerons seulement quelques faits. Nous apprenons le sens de mots étrusques à partir de gloses (qui peuvent tout aussi bien indiquer le sens d'un mot étrusque en $\varepsilon \vee$ 
grec). Certains témoignages nous livrent des étymologies : c'est le cas pour Tite-Live et d'autres auteurs qui nous apprennent, par exemple, que le terme latin histrio servant à désigner l'acteur vient de l'étrusque ister $^{37}$. D'autres proviennent de «dictionnaires", comme le De significatu uerborum de l'érudit augustéen Verrius Flaccus, qui fut abrégé au II ${ }^{\text {ème }}$ ou III ${ }^{\text {ème }}$ après J.C. par Sextus Pompeius Festus (le lexique de Festus fut lui-même abrégé au VIII ${ }^{\text {ème }}$ siècle par paul Diacre et ne nous est parvenu que de façon incomplète) : c'est le cas pour les termes nepos ou subulo. Ces gloses nous apprennent aussi, par exemple, que acale signifie «juin», celi signifie « septembre ». Certaines apparaissent dans des études tardives : c'est dans les Étymologies d'Isidore de Séville (560-636) que les mots latins cassis et lanista nous sont présentés comme d'origine étrusque ${ }^{38}$.

Ce peut être aussi de façon détournée, au hasard d'un récit étiologique, que nous découvrons un mot étrusque: Suétone ${ }^{39}$ et Dion Cassius $^{40}$ racontent comment un prodige qui se produisit peu avant la mort d'Auguste annonça sa prochaine divinisation: la foudre tomba sur l'inscription d'une statue à son effigie où figurait son nom complet, Caesar Imperator Augustus, juste après le $« \mathrm{C} »$ de Caesar, ce que les devins interprétèrent comme le passage de l'imperator Augustus dans le monde des dieux, aesar (aisar) en étrusque, cent (C) jours plus tard.

Cependant, la fiabilité des informations apportées par ces gloses peut parfois laisser à désirer, au regard des données épigraphiques ou archéologiques ; ainsi, la tradition latine affirme que Lucumon (le nom porté par le futur Tarquin l'ancien avant son arrivée à Rome) est un mot étrusque qui signifie «roi »; or, l'existence fréquente de formes apparentées à ce nom dans l'onomastique étrusque fait plutôt concevoir l'hypothèse d'un anthroponyme auquel les antiquaires romains auraient attribué artificiellement cette signification, justement à partir de la tradition romaine sur Lucumon devenu le roi Tarquin.

Varron reconnaissait volontiers que la langue latine avait puisé dans le vocabulaire étrusque ${ }^{41}$, mais, en réalité, les gloses de nos sources anciennes ne nous livrent tout au plus qu'une soixantaine de mots de cette langue. Quant à la trentaine de bilingues étrusco-latines, toutes de datation tardive et limitées à l'apport onomastique que permet leur nature funéraire, elles ne traduisent que la volonté de faire comprendre dans la langue désormais irrémédiablement dominante, le latin, des épitaphes traditionnellement formulées dans la langue des ancêtres ${ }^{42}$. Il faut donc bien constater un manque d'intérêt relatif pour la langue étrusque, malgré le sursaut du tournant du $\mathrm{I}^{\mathrm{er}}$ siècle, ce qui explique les difficultés de compréhension qui se 
poseront à la Renaissance et, par la suite, à tous ceux qui voudront exhumer la langue des anciens Toscans.

\section{Traduire l'étrusque à la Renaissance}

Il est remarquable que les fausses explications étymologiques se retrouveront à la base des tentatives modernes de traduction de l'étrusque alors que la Renaissance, avec le monde gréco-romain et ses textes, découvrait aussi les anciens Toscans. Nous prendrons deux figures empruntées à cette période et liées étroitement à la redécouverte des Étrusques.

La première est celle d'Annius de Viterbe (Giovanni Nanni), frère dominicain qui vécut de 1432 à 1502 . Il était professeur de théologie, mais n'hésitait pas à se tourner vers la Kabbale juive et ce qu'on savait de l'ancien Orient; Annius s'était passionné en particulier pour les antiques habitants de la province de Viterbe, dans le Latium actuel, qui était une ancienne cité étrusque. Il en découvrait la trace chez les auteurs classiques et il les réintégra dans la reconstitution de l'histoire de l'Antiquité - et du monde - qu'il publia en 1498 à Rome sous le titre de Commentaire du frère Jean Annius de Viterbe, de l'ordre des frères prêcheurs, professeur de théologie, au sujet des ouvres de divers auteurs parlant des Antiquités ${ }^{43}$. Ces Antiquités, (nom sous lequel l'œuvre est généralement connue), eurent un énorme succès : plusieurs rééditions parurent, dont deux à Paris dès 1512 et 1515 .

De fait, le contenu de ces Antiquités ne nous paraît guère scientifique aujourd'hui, car Annius se lançait dans un mélange étonnant de traditions juives et d'antiquités classiques ; par exemple, il identifiait Noé au dieu Janus par une simple analogie : ce dernier avait jadis abordé en Italie sur un navire ; cela suffisait au dominicain pour dire qu'il s'agissait de l'arche du patriarche, qui, rescapé du Déluge, venait fonder la civilisation étrusque ! $\mathrm{Ou}$ encore, il rapprochait l'Etrusca disciplina, considérée d'après le témoignage de Pline l'Ancien, de la kabbale ${ }^{44}$. En outre, Annius appuyait fréquemment ses démonstrations sur de pseudo-citations de divers auteurs antiques - toujours assez imprécises pour qu'on ne pût trop facilement vérifier leur véracité : au nombre des fragments d'œuvres anciennes qu'il prétendait avoir retrouvées et qu'il publia, on peut citer, notamment, celle du prêtre chaldéen Bérose dont la tradition ne nous a livré que quelques fragments (en particulier chez Flavius Josèphe): Annius n'hésitait pas à élaborer des faux à partir de ces fragments authentiques et de versets de la 
Genèse revus et complétés par ses soins ${ }^{45}$. Le «faux-Bérose » fut largement utilisé avant que l'on ne découvre la supercherie.

Même si cette démarche est scientifiquement condamnable, elle avait toutefois le mérite - au moment où la redécouverte de l'Antiquité classique se concentrait sur la Grèce et Rome et risquait de laisser de côté toutes les autres grandes civilisations - d'exhumer l'Étrurie et d'en amorcer une résurrection. D'ailleurs, le théologien de Viterbe se montrait d'autant plus original pour son époque qu'il mettait entre parenthèses la civilisation gréco-romaine en liant directement les Étrusques à la tradition hébraïque et aux origines du monde telles que les racontait la Genèse. Les Étrusques acquéraient donc une ancienneté plus que respectable puisque, remontant au Déluge, ils étaient en quelque sorte le nouveau peuple élu. Toute cette reconstruction reposait sur le fait qu'Annius voyait dans l'étrusque une langue apparentée à l'hébreu.

Cette vision pour le moins originale allait orienter considérablement les tentatives de traduction de l'étrusque à la Renaissance, d'autant que la reconstruction historique qu'elle soutenait n'était pas qu'une simple spéculation de savant : elle intéressait le pouvoir. Les auteurs du Trecento ne l'avaient certes pas attendue pour utiliser politiquement le mythe étrusque. Des savants comme G. Villani, C. Salutati et L. Bruni ne s'en étaient pas privés : les deux premiers pour justifier l'idée d'une unification de la Toscane, sous la direction de Florence ${ }^{46}$; le dernier pour convaincre Gonzague de Mantoue, si ce n'est d'entrer dans une confédération florentine, du moins de ne pas tomber sous la coupe de Milan, rivale de Florence depuis la guerre de $1390-1402^{47}$.

Cependant, à partir des travaux d'Annius, ceux qui se considéraient comme les légitimes continuateurs des souverains de la Toscane antique, les Médicis, encouragèrent systématiquement les recherches qui tendaient à exhumer le passé prestigieux de la région. Ce fut surtout l'œuvre de Laurent le magnifique (1449-1492) et, encore plus, de Côme $1^{\text {er }}(1519-1574)$ qui, le premier, reçut le titre de grand-duc de Toscane. Ce dernier revendiqua explicitement l'héritage des antiques lucumons et plus précisément de Porsenna, le fameux roi de Chiusi. La propagande ducale en livre un exemple éclatant : le décor allégorique prévu pour les noces du quatrième fils de Côme $1^{\mathrm{er}}$, Ferdinand $1^{\mathrm{er}}$, avec Christine de Lorraine en 1589, établissait un lien direct entre Porsenna et Côme de Médicis, puisque la Toscane y était représentée, couronnée, sous leur double figure, avec l'inscription «Diadème du roi Porsenna, perdu par la négligence et récupéré par la vertu et la diligence de Côme de Médicis ». 
Dans ces circonstances, on comprend que Côme fut un grand amateur et collectionneur d'antiquités étrusques : c'est lui qui acheta la célèbre Chimère d'Arezzo (pièce fabriquée vers 400 avant J.-C.), trouvée à l'automne 1553 alors qu'on travaillait à la construction de la muraille de la ville ; elle fut amenée à Florence, exposée au Palazzo Vecchio où Benvenuto Cellini la restaura. Le goût pour les Étrusques de Côme et son souci de propagande personnelle l'amenèrent à fonder en 1541 une Académie florentine, dont les recherches sur les anciens Toscans étaient l'un des thèmes principaux. On y suivait les idées d'Annius de Viterbe et y étudiait l'hypothèse d'une langue « aramaïque » parlée par Noé et ses descendants, en Italie comme au ProcheOrient. Cette parenté de la langue étrusque avec l'hébreu se retrouve dans des œuvres comme le Dell'origine di Firenze de Giovan Battista Gelli (1544) et l'Il Gello de Pier Francesco Giambullari, l'un des fondateurs de l'Académie, $(1546)^{48}$.

C'est dans la tradition de ces écrivains florentins du Cinquecento, qui entretinrent le mythe de l'origine aramaïque des Étrusques inspiré par Annius de Viterbe et lui donnèrent une lecture politique, qu'il faut placer le Français Guillaume Postel, seconde figure qui retient ici notre attention ${ }^{49}$. L'intérêt de celui-ci pour les Étrusques tient à ses compétences linguistiques considérables pour son époque, mais il est aussi à comprendre dans la perspective des liens culturels et génétiques entre Orient et Occident que ce savant voulut mettre en évidence, ainsi que du point de vue des conceptions politiques et eschatologiques qui ont marqué son orientalisme.

Guillaume Postel mena une vie si remplie et mouvementée qu'il convient d'en rappeler les grandes lignes pour mieux comprendre ses rapports avec l'Orient et l'origine de son intérêt pour les Étrusques et leur langue ${ }^{50}$.

Cet humaniste, grand voyageur et étonnant autodidacte, est né en 1510 près de Barenton en Normandie ; ayant perdu ses parents morts de la peste alors qu'il était encore tout jeune, il s'instruisit si vite qu'il devint maître d'école à l'âge de treize ans. Vivant comme domestique à Paris, Amiens ou Rouen, il s'initia seul à l'hébreu avec un alphabet, une grammaire et un exemplaire latin-hébreu des Psaumes. L'un des nombreux protecteurs que son intelligence lui valut, Jean Raguier, abbé d'Arras, en fit le précepteur de son neveu à Paris, l'ambassadeur Jean de la Forest, qu'il accompagna et fréquenta quelques mois à Constantinople en 1535-1536, au moment où François I ${ }^{\text {er }}$ négociait avec Soliman le Magnifique l'alliance des Ottomans contre Charles-Quint. Postel partait pour découvrir des manuscrits anciens 
pour la collection du palais de Fontainebleau. En 1538, le roi reconnut son génie et le nomma lecteur pour l'enseignement du grec, de l'hébreu, de l'arabe - puis des mathématiques - dans le Collège royal qu'il venait de fonder à l'instigation de Guillaume Budé (l'actuel Collège de France) ${ }^{51}$. C'est alors que Postel publie ce que l'on peut considérer comme une première grammaire comparée avec son ouvrage sur Les douze langues différant par les caractères de leurs alphabets ${ }^{52}$.

En 1542, son protecteur et ami le chancelier Poyet tombe en disgrâce ; son intercession en sa faveur à la cour le met en délicatesse avec le pouvoir et Postel finit par quitter la France pour Rome où il arrive en mars 1544.

Lors de son séjour en Italie, Postel fut conquis par Ignace de Loyola qui venait de fonder l'ordre des Jésuites, si bien qu'il entra au noviciat de la compagnie de Jésus et fut ordonné prêtre. Mais les idées aventureuses de cet homme déjà empli de rêves messianiques inquiétèrent le saint qui vit en lui quelqu'un qui voulait faire de la Compagnie l'instrument de ses propres idées : il l'exclut de sa congrégation ${ }^{53}$. Emprisonné comme hérétique, Postel s'enfuit de Rome et se réfugia à Venise, où il fut aumônier de l'hôpital Saint-Jean et Saint-Paul. C'est là qu'il fit la connaissance, en 1547, de la Mère Jeanne, une sainte femme quelque peu visionnaire, qui bouleversa sa vie en lui dévoilant son destin prophétique. Il finit par être dénoncé à l'Inquisition, qui, heureusement pour lui, le jugea plus fou qu'hérétique.

En août 1549 il fuit donc Venise, embarque avec des pèlerins pour Jérusalem, où il séjourne et se lie avec André Thevet, qui accompagnait l'ambassadeur Gabriel de Luetz, baron d'Aramont ; c'est là qu'il rencontre aussi Jean Chesneau et Pierre Gilles, deux autres «chasseurs de manuscrits » en mission pour François $\mathrm{I}^{\mathrm{er}}$, avec lesquels il revint par Constantinople. Lors de ce nouveau séjour en Orient, l'ambassadeur d'Aramont lui permet d'acquérir quelques manuscrits rares ${ }^{54}$. Ces voyages amènent Postel à étudier la religion des Syriens et des chrétiens maronites. Il émet l'hypothèse que les Druses, à la fois musulmans, chrétiens et païens, étaient les descendants des Gaulois (les Galates d'Orient) et que leur nom venait de Dreux et de druides.

Postel revint à Paris en 1552-1553, en passant par la Suisse (Bâle et Genève). Il rapporta quelques manuscrits orientaux à la bibliothèque royale de Fontainebleau. Mais la publication d'un premier livre dans lequel il proclamait la Mère Jeanne - issue pour lui de la substance de Jésus-Christ seconde Ève annonciatrice des temps nouveaux, déclenche un scandale qui lui vaut de reprendre les routes de 1'exi1 ${ }^{55}$ : Besançon, Bâle, Venise... Nommé professeur de mathématiques par Ferdinand I ${ }^{\text {er }}$, roi des Romains, il 
reste à Vienne deux ou trois ans, revient à Venise où il publie la Vierge vénitienne, toujours sur la Mère Jeanne. Impécunieux, il loue ses précieux manuscrits au duc de Bavière et circule encore beaucoup en Italie (Pavie, Crémone, Padoue). Postel est de nouveau emprisonné pour ses écrits « hérétiques » à Ravenne puis à Rome en 1555 et ne sera libéré qu'à la mort de Paul IV. Le savant retourne finalement à Paris où il s'établira définitivement en 1562, non sans être repassé, entre autres villes, à Venise, en 1561, pour la vente de ses manuscrits, et à Trente pour essayer de voir et d'influencer les Pères du Concile.

Bien qu'il ait rétracté quelques-unes de ses opinions audacieuses (ou plus exactement donné une explication admissible à ses théories sortant de l'ordinaire), il fut enfermé au monastère de Saint-Martin-des-Champs par arrêt du Parlement. Il y vécut de façon relativement libre puisqu'il continuait à donner ses cours avec succès, à recevoir les plus grands personnages - savants, lettrés et même le roi Henri III ; il pouvait au demeurant sortir quand il le voulait. Ce savant respecté, quoiqu'original, mourut le 6 septembre 1581 .

Entré en contact direct avec l'Orient dès 1535, Postel semble avoir été le premier à comprendre l'importance scientifique de l'orientalisme et à pressentir la future coopération des nations musulmanes et de l'Europe, l'un des premiers à voir la grandeur des civilisations orientales et des liens génétiques avec la civilisation européenne. Sa nomination au collège royal fit aussi de lui un précurseur dans l'enseignement des langues orientales, anciennes et modernes, et des littératures qu'elles véhiculaient ${ }^{56}$. On lui doit notamment d'avoir fait découvrir à l'Europe l'historien et géographe arabe Ab'ul-Fida. De même, alors que le Coran était complètement ignoré ${ }^{57}$, Postel le fit connaître : c'était pour ce savant et homme de foi non seulement un moyen d'éclairer le christianisme, mais encore de réconcilier les deux religions.

On comprend dès lors qu'un esprit si ouvert se soit intéressé aux travaux d'Annius de Viterbe : l'hébraïsant Postel, de même qu'il avait inventé de toutes pièces l'origine samaritaine des Turcs, suivait de même sans problème les inventions d'Annius en ce qui concernait les Étrusques.

Postel reprend donc dans son De Etruriae regionis... originibus, institutis, religione et moribus... ${ }^{58}$, paru en 1551, l'idée que les Étrusques, en Italie, tout comme les Hébreux, au Proche-Orient, parlaient une langue apparentée descendant de la langue de leur ancêtre commun, Noé (Noachus). Ils seraient en effet arrivés d'Assyrie après le Déluge, guidé par or 
un chef, duquel ils tiraient leur savoir et leur religion. Ce peuple originaire d'Aturie ou d'Éturie, avant que le nom originel ne fût corrompu en Etruria, avait reçu ses institutions religieuses d'un certain Janus, chef de la province appelée Tuscia. ${ }^{59}$ Postel ne se borne pas à rendre compte du doublet Tuscia/Etruria dans la dénomination de l'Étrurie dont il pose les origines proche-orientales ou à donner le nom du grand dieu italique et romain à ce prince éturien: superposant légendes antiques et Ancien Testament, Postel fait agir Janus strictement comme le patriarche biblique face au Déluge :

"Secundi enim mensis die decima septima incoepit pluuia et

Ianus cum tota domu intrauit in arcam in salutem generis humani comparatam $\gg .60$

L'identification de Janus avec Noé est parfaite, mais il ne connaît pas le même sort que le fils de Lamech ou que l'Outa-Napishtim sumérien. C'est en Italie qu'il débarque, y introduisant les lettres et institutions de ses ancêtres orientaux. ${ }^{61}$ Ce personnage de Noachus-Janus, qui apportait donc la race, la langue et la civilisation étrusque en Italie, permettait à Postel de faire ressortir la haute antiquité et l'illustre origine des anciens Toscans tout en resserrant les liens entre Orient et Occident.

Les développements du De Etruriae... vont plus loin. Noé-Janus diffusait en Étrurie - par l'intermédiaire de ses fils, les mythiques Razi Hinui, prêtres mystiques - des connaissances et pratiques diverses - dans les domaines religieux et agricole, par exemple -, et, surtout, il établissait les principes d'un ordre universel et éternel préfigurant l'avènement du Christ. De ce fait, la tradition sur les duodecim populi de la nation étrusque, dont Postel attribue la création à Janus ${ }^{62}$, est à lire selon une perspective religieuse : le caractère sacré de ce nombre 12 est pour l'humaniste la preuve formelle de l'essence divine et métaphysique du peuple étrusque et il ne manque pas de mettre en rapport les douze peuples de l'Étrurie avec les douze tribus d'Israël et, dans la perspective chrétienne, avec les douze apôtres. Une autre preuve en est, pour Postel, l'appétence toute particulière de ce peuple pour les choses de la religion et sa maitrise de pratiques divinatoires variées. Reprenant la tradition romaine qui présente les Étrusques comme la nation plus que tout autre dédiée aux choses de la religion ${ }^{63}$, Postel tire argument de la place de la disciplina Etrusca à Rome et de la pratique des élites romaines de faire instruire leurs fils en Étrurie (pratique que nous avons commentée précédemment) pour avancer le rôle missionnaire des Étrusques: ils ont préparé un terrain propre à accueillir le christianisme ${ }^{64}$. Le symbole le plus fort en serait la crucifixion de Saint Pierre là même où avait été enseveli NoéJanus $!^{65}$ 
Le primat culturel et religieux que Postel accordait aux Étrusques débouchait naturellement, comme l'ont bien montré G. Cipriani et C. Vasoli ${ }^{66}$, sur le constat qu'après le long triomphe de Rome était venu le temps des nouveaux Toscans, des héritiers directs et légitimes des Étrusques, de ces fils de Janus, et en particulier des Florentins. C. Vasoli a supposé un projet politique habilement caché derrière le réseau étroit des mythes, allusions et symboles qui font la texture du De Etruriae... ${ }^{67}$ Mais par delà ces judicieuses analyses qui tiennent compte de l'activité politique française qui reprend alors essentiellement en direction de Sienne -, des menées de la cour florentine face à cette ambitieuse rivale, de l'existence d'un milieu francophile à Florence ou, plus prosaïquement, du souci de propagande personnelle de Côme, la motivation profonde de Postel est très certainement à chercher du côté de ses propres perspectives eschatologiques et de sa grande théorie de la Restitution ${ }^{68}$.

Si Postel donna donc une impulsion féconde aux études orientales, dont il comprit l'avenir scientifique, et à l'étude des langues, sa vision des Étrusques et son approche de leur langue furent limitées : en perpétuant le mythe étrusque qui s'était développé en Italie et en inscrivant dans un grand dessein général son intérêt pour les Étrusques, ce savant, malgré une originale monographie consacré aux anciens Toscans, sclérosait en quelque sorte tout progrès sur la compréhension d'une langue déjà morte depuis longtemps.

\section{Conclusion}

Les tentatives de traduction de l'étrusque par Annius et Postel visaient manifestement à utiliser les Étrusques et la redécouverte de leur langue pour légitimer les interactions entre les cultures de leur temps tout en les inscrivant dans la perspective de l'héritage antique.

L'époque contemporaine a vu, fort heureusement, s'effacer progressivement le détournement de la langue et de la civilisation étrusques pour se concentrer sur des problèmes de traduction plus scientifique ; cela ne s'est cependant pas fait sans errements.

Quelques essais de rapprochements de l'étrusque avec une autre langue, qui sont pourtant le fait de savants reconnus, nous paraissent aujourd'hui tout aussi hasardeux : W. Corssen, en 1874-1875, faisait de l'étrusque une langue italique dans son Über die Sprache der Etrusker, donc proche du latin du Latium, de l'ombrien de l'Ombrie, du sabin de Sabine, de 00 
l'osque de Campanie et du Samnium, alors que l'on rejette désormais le caractère indo-européen de l'étrusque. V. Thomsen, dans ses Remarques sur la parenté de la langue étrusque publiées à Copenhague en 1899, rapprochait l'étrusque des langues caucasiennes; J. Martha, La langue étrusque, 1913, en faisait une langue finno-ougrienne; plus récement, V. Georgiev le rapprochait encore d'une langue indo-européenne, le hittite ${ }^{69}$. Ces tentatives de rapprochement forcé avec une autre langue sont désormais abandonnées et l'on privilégie d'autres méthodes de traduction (bilingustique, etc). Mais force est de constater que la perte des traductions latines de l'étrusque a considérablement nui à l'histoire de l'étruscologie et à une meilleure connaissance de la civilisation des Étrusques et de leurs échanges avec leurs contemporains.

Ce qui se passe entre l'étrusque et le latin est bien la preuve que, sans le souci de la traduction et de la pérennisation des savoirs et des savoir-faire qu'elle implique, l'interaction entre les cultures ne peut plus être pleinement perçue. Un grand esprit et un travailleur infatigable comme Cicéron avait compris l'importance de donner aux lettres latines tout le vocabulaire qui lui manquait, notamment dans le domaine de la rhétorique et de la philosophie ; il est dommage que nous n'ayons pas eu de travail équivalent en langue étrusque ou, s'il a jamais existé, qu'il se soit perdu. 
Notas

${ }^{1}$ La forme ancienne tevara $\theta$ apparaît deux fois sur la tombe des Augures de Tarquinia (VI ${ }^{\text {ème }}$ siècle avant J.-C.) à côté d'un personnage qui, tenant un lituus à la main, règle un combat (CIE 5331 et 5332 ; Rix Ta 7.7 et 7.8).

${ }^{2}$ Tabula Cortonensis, [a cura di] Luciano Agostiniani, Francesco Nicosia, Roma, L'Erma di Bretschneider, 2000.

${ }^{3}$ Notons toutefois que cette interprétation de la table de Cortone comme un document juridique ne fait pas l'unanimité : C. De Simone y voit un texte religieux décrivant une parentatio, cérémonie funéraire dans «La Tabula Cortonensis : tra linguistica e storia », Annali della Scuola Normale Superiore di Pisa, 4, 3, 1998 (2000), p. 1-122. Et d'autres lectures et interprétations ont été avancées depuis : voir notamment Facchetti (Giulio M.) Frammenti di diritto privato etrusco. Firenze Leo S. Olschki, 2000 ; Maristella Pandolfini e Adriano Maggiani (ed.), La Tabula cortonensis e il suo contesto storicoarcheologico : atti dell'incontro di studio, 22 giugno 2001, Roma, Consiglio Nazionale delle Ricerche, 2002; Scarano Ussani (Vincenzo)-Torelli (Mario), La «Tabula Cortonensis » : un documento giuridico, storico e sociale, Napoli, Loffredo, 2003.

Sur cette formule étrusque, voir Lambrechts (Roger), Essai sur les magistratures des

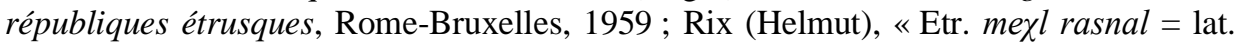
res publica », Studi di antichità in onore di G. Maetzke, Rome, 1984, p. 455-468.

${ }^{4} \mathrm{~K}$. Olzscha, «Götterformeln und Monatsdaten in der grossen etruskischen Inschrift von Capua” Glotta, 34, 1955, p. 71-93.

${ }^{5}$ H. Rix, «Inhalt und Funktion des Textes auf der Tontafel von Capua », actes du Colloque Die Welt der Etrusker, sous la direction de H. Heres et M. Kunze, Berlin, 1988 (1990), p. 111-115.

${ }^{6}$ M. Cristofani, Tabula Capuana, un calendario festivo di età arcaica, Biblioteca di Studi Etruschi, 29, Florence, Leo S. Olschki, 1995.

${ }^{7}$ Section 1, lignes 3-4 : le Oamsul cim tartiria cim cleva acasri ; cf. Cristofani 1995, p. 57.

${ }^{8}$ Tite-Live IV, 7, $12 ; 13,7 ; 20,8$.

9 F. Roncalli, «Osservazioni sui libri lintei etruschi», Rendiconti della Pontificia Accademia di Archeologia, 51-52, 1978-1980, p. 23-21 et, id., «Carbasinis voluminibus implicati libri, osservazioni sul liber linteus di Zagabria », Jahrbuch des deutschen archäologischen Instituts, 95, 1980, p. 227-264. C'est le cas sur un sarcophage de la Tombe des Sarcophages de Cerveteri, conservé au Musée Grégorien du Vatican. On pense aussi reconnaître ces livres dans un décor de la tombe des Reliefs de Caeré. Nous connaissons par ailleurs l'existence de livres étrusques sur parchemin : un bon exemple en est fourni par la sépulture de Lars Pulenas à Tarquinia, dont le gisant déroule devant lui un uolumen.

${ }^{10}$ Il serait vain de vouloir identifier les circonstances précises de l'arrivée de ce document : avec les troupes envoyées en Égypte par Pompée sous les ordres de Gabinius ? Lors de la campagne Alexandrine de César? Au cours de l'aventure orientale d'Antoine ? Au moment de l'annexion par Auguste?

Sur l'Etrusca disciplina voir C. O. Thulin, Die Etruskische Disciplin, Göteborg, 1905-1909. En français, synthèse récente de J.-R. Jannot, Devins, dieux et démons, Regards sur la religion de l'Étrurie antique, Paris, Picard, 1998.

${ }^{11}$ J. Heurgon, La Vie quotidienne chez les Étrusques, Paris, 1961, p. 297. 


\section{Traduire l'étrusque : problématiques antiques et modernes}

12 ap. Diodore de Sicile, BH, V, 40 (= FGrH 87 F 119, Theiler 83).

13 Varron, De lingua latina $(\mathrm{V}, 55)$. Il s'agit ici de M. Iunius Gracchanus, ami de C. Gracchus et historien du droit public.

${ }^{14}$ Nous empruntons cette traduction à J. Collart, Varron. De lingua latina, V, Paris, Les Belles-Lettres, 1954.

${ }^{15}$ J. Heurgon, La Vie quotidienne chez les Étrusques, Paris, 1961, p. 304.

${ }^{16}$ J. Heurgon, «Un témoignage de Plaute sur la tragédie étrusque », dans Mélanges Jean Bayet (collection Latomus 70), Bruxelles, p. 260-263 = Scripta varia (collection Latomus 191), Bruxelles, 1986, p. 415-418. C'est le nom que Plaute accole au summus imperator Bumbomachides au vers 14 du Miles.

${ }^{17}$ F.-H. Massa-Pairault, Recherches sur l'art et l'artisanat étrusco-italiques à l'époque hellénistique, Rome, EFR, 1985.

${ }^{18}$ Ce monument retrouvé par G. Maetzke est du milieu du II ${ }^{\text {ème }}$ siècle. Sur les structures du théâtre en Étrurie, voir G. Colonna, «Strutture teatriformi in Etruria», dans J.P.Thuillier (éd.), Spectacles sportifs et scéniques dans le monde étrusco-italique, Colloque Rome 1991, (CEFR 172), Rome, 1993, p. 321-347.

${ }^{19}$ D. Briquel, « À la recherche de la tragédie étrusque », Pallas, 1998, 49, p. 35-51.

${ }^{20}$ Varron, ap. Censorinus, De die natali, 17, 6.

${ }^{21}$ D. Briquel, «Claude, érudit et empereur », CRAI, 1988, p. 217-232.

${ }^{22}$ Suétone, Cl., 42, 5. Sur cet ouvrage perdu, voir D. Briquel, «Que savons-nous des Tyrrhènika de l'empereur Claude ? », RFIC, 116, 1988, p. 448-470 et $i d$., «L'empereur Claude comme auteur des «Tyrrhenika » », dans Les écrivains et l'Etrusca disciplina de Claude à Trajan, Caesarodunum supplément 64, 1995, p. 88-93.

${ }^{23}$ J. Heurgon, «La vocation étruscologique de l'empereur Claude », CRAI, 1953, p. 93-97.

${ }^{24}$ CIL, XIII, 1668 ; voir P. Fabia, La Table claudienne de Lyon, Lyon, 1929. Ce discours a été réécrit par Tacite dans ses Annales, XI, 24.

25 Voir sur ce point D. Briquel, Le regard des autres, Besançon, Annales Littéraires de l'Université de Franche-Comté, n ${ }^{\circ} 623,1997$, p. 57-116.

${ }^{26}$ Tacite, Ann., XI, 15. Avec la volonté de faire ainsi barrage aux religions orientales (v. sur ce point J. Heurgon « Tarquitius Priscus et l'organisation de l'ordre des haruspices sous l'empereur Claude», Latomus 12, 1953, p. 402-415 ; D. Briquel, ibid. p. 101 sq.); l'Etrusca disciplina y contribue à maintenir le mos maiorum.

${ }^{27}$ Sur les elogia Tarquiniensia et les exploits d'un Spurinna au V ${ }^{\text {ème }}$ ou au IV ${ }^{\text {ème }}$ siècle, voir en particulier M. Torelli, Elogia Tarquiniensia, Rome, 1975 et G. Colonna, «Apollon, les Étrusques et Lipara », MEFRA, 96, 1984, 2, p. 557-578.

${ }^{28} \mathrm{~V}, 1,6$ : gens itaque ante omnes alias eo magis dedita religionibus quod excelleret arte colendi eas ...

${ }^{29} \mathrm{Ce}$ signe, dans l'alphabet latin, prend la place du $\mathrm{Z}$ étrusque transcrivant le son [ts] que le latin pouvait noter par le digramme TS.

${ }^{30} \mathrm{IX}, 36,3-4$.

${ }^{31}$ J. Heurgon ibid., p. 294-296

${ }^{32}$ Le dossier sur les liens entre Caeré et Rome est considérable : rappelons ici seulement l'ouvrage fondamental de M. Sordi, I rapporti Romano-ceriti e l'origine della civitas sine suffragio, Rome, 1960. 
${ }^{33}$ Inscription du troisième quart du VII ${ }^{\text {ème }}$ siècle sur un vase : mi kalaturus papenas cenecu hetie ; Rix Cr. 2.31.

34 J. Heurgon, «Varron et l'haruspice étrusque Tarquitius Priscus », in J. Collart (éd.), Varron, grammaire antique et stylistique latine, Paris, Les Belles Lettres, 1978, p. 101104.

35 A. Piganiol, «Le calendrier brontoscopique conservé dans le De ostentis de Jean Lydus », REL 1949 , p. 84-85 et id., « Sur le calendrier brontoscopique de Nigidius Figulus », Stud. in honor of A. Ch. Johnson, p. 79-87.

36 G. Breyer, Etruskisches Sprachgut im Lateinischen unter Ausschluss des spezifisch onomastischen Bereiches, Leuven : Peeters, 1993.

${ }^{37}$ IX, 2, 6-7.

38 D. Briquel, «Deux mots latins d'origine étrusque selon Isidore de Séville», Moussylanea: mélanges de linguistique et de littérature anciennes offerts à Claude Moussy, Bruno Bureau, et Christian Nicolas (éd.) Louvain-Paris, Peeters, 1998, p. 3739.

39 Vie d'Auguste, 47.

${ }^{40}$ Histoire romaine, 56, 29, 4.

${ }^{41}$ Voir J. Collart, Varron, p. 243 sq.

42 Sur ces documents, voir désormais E. Benelli, Le iscrizioni bilingui etrusco-latine, Firenze, Olschki, 1994 et J. Hadas-Lebel, Le bilinguisme étrusco-latin : contribution à l'étude de la romanisation de l'Étrurie, Leuven, Peeters, 2004.

43 Commentaria fratris Ioannis Annii Viterbensis, Ordinis Praedicatorum, theologiae professoris super opera diuersorum auctorum de Antiquitatibus loquentium, Romae, in Campo Florae, anno Domini MCCCCXCVIII (Florence, Biblioteca Marucelliana).

44 «Plinius tradit in disciplina etrusca contineri quo pacto possint euocari dii, fulgura, ignes et eiuscemodi. Quare etiam quia oppositorum est eadem disciplina, rituales continebant, quo pacto non possint euocari. Hoc autem nullo modo fieri melius poterat quam occultando nomen ipsius Dei, in cuius tutela urbs ipsa erat. Eius occultandi modus erat Etruscis, mysterio quodam, et literis quibusdam ab ipso dei nomine alterum extrahere, cuius ritu ac mysterio nunc soli Talmudistae Caballarii utuntur in disciplinam quam uocant Cabala. »

Antiquitates, édition de 1552, p. 580.

45 Il citait de même l'Égyptien Manéthon, le Perse Mégasthène ou encore Myrsile de Lesbos.

${ }^{46}$ G. Villani, Cronica, in Croniche di Giovanni, Matteo e Filippo Villani secondo le migliori stampe e corredate di note filologiche e storiche, Trieste-Milano, lloyd Austriaco 1857 ; C. Salutati, Lettre aux Pérugins du 19 juillet 1383 et Invective contre Loschi de 1399.

47 Lettre 25 in Leonardi Bruni Arretini epistolarum libri VIII... recensente Laurentio Mehus, Florence, 1741, livre X, pp. 217-229. Bruni y insiste sur l'origine purement étrusque de Mantoue (cf. Virg., En. X, 198-199). Sur cette lettre, voir L. Pradelle, « La Lettre sur l'origine de Mantoue de Leonardo Bruni : le mythe étrusque pour une conquête politique », in B. Westphal (éd.), Le rivage des mythes. Une géocritique méditerranéenne : le lieu et son mythe, Limoges, PULIM, 2001, pp. 179-193.

${ }^{48}$ Ces ouvrages, qui avaient pour but de consolider le principat des Médicis, découlent pour ○q une large part de la politique de Côme face aux ambitions de Sienne. Voir sur ce point 


\section{Traduire l'étrusque : problématiques antiques et modernes}

M. Plaisance, « Culture et politique à Florence de 1542 à 1551 : Lasca et les « Humidi » aux prises avec l'Académie Florentine », Les écrivains et le pouvoir en Italie à l'époque de la Renaissance (deuxième série), A. Rochon (éd.), Paris, 1974, p. 149-242.

Dans Il Gello, où il prouve son intérêt pour les lettres hébraïques, Giambullari (1495-1564) veut montrer que la langue italienne venait de l'ancien étrusque en se servant de l'hébreu et de l'araméen. Cet ouvrage est un hommage à son compatriote et collègue G. B. Gelli (1498-1563), qui joue un rôle important dans le dialogue pour montrer que « la maggior parte de nostri nomi non dependono dal Latino ma dell'Etrusco : il quale certamente si puo male intendere ne tempi nostri senza un ottime di queste altre lingie, della Hebrea dico et della Caldea, lequali uscirono donde la Etrusca ».

49 Voir sur ce point C. Vasoli, «Postel e il 'Mito etrusco'», Mélanges à la mémoire de Franco Simone. France et Italie dans la culture européenne. I Moyen-âge et Renaissance, Genève, Slatkine, 1980, p. 325-350.

${ }^{50}$ Sur la biographie détaillée de Guillaume Postel, on consultera De Guglielmi Postelli vita et indole de G. Weill, Paris, Hachette, 1892, étude traduite du latin par J. Parasié et F. Secret et mise à jour par F. Secret, Vie et caractère de Guillaume Postel, Milan, Archè, 1987, avec bibliographie complémentaire p. 23-28. Pour s'en tenir à quelques études récentes, citons : W. J. Bouwsma, Concordia mundi : The Career and Thought of Guillaume Postel (1510-1581), Cambridge (Mass.), Harvard University Press, 1957 (qui ne prend en compte que la production imprimée de Postel) ; F. Secret, Les kabbalistes chrétiens de la Renaissance, Paris, Dunod, 1964, p. 171 sq. ; Id., Notes sur Guillaume Postel, «Bibliothèque d'Humanisme et Renaissance, XXI, 1959, p. 461 sq. ; M. L. Kuntz, Guillaume Postel Prophet of the Restitution of All Things. His Life and Thought, The Hague - Boston - London, 1981. La liste des travaux dédiés par F. Secret à Postel sont recensés dans F. Secret, Bibliographie des manuscrits de Guillaume Postel, Genève, Droz (Paris, Minard), 1970, p. 147-151. Sur l'œuvre de Postel, voir, plus récemment, l'ouvrage de Claude Postel, Les écrits de Guillaume Postel publiés en France et leurs éditeurs 1538-1579, Droz, Travaux d'Humanisme et Renaissance CCLXV, 1992. Cet ouvrage présente aussi une recension des travaux modernes sur G. Postel, p. 998 -106. Enfin, une approche éclairante de l'histoire subjective de Guillaume Postel est aussi donnée par J. Simmonnet, «Guillaume Postel : Rêve, Délire, Illumination? », L'Information psychiatrique, vol. 55, n 1 (janvier 1979), p. 53-64.

${ }^{51}$ Cf. A. Lefranc, Histoire du Collège de France depuis ses origines jusqu'à la fin du premier Empire, Paris, 1893.

52 Linguarum duodecim characteribus differentium alphabetum, introductio, ac legendi modus longe facillimus. Linguarum nomina sequens proxime pagella offeret. G.P., publié à Paris en 1538.

538 décembre 1545.

${ }^{54}$ Sur le détail de ce second voyage en Orient, voir M. L. Kuntz, op. cit., 1981, p. 93-99.

${ }^{55}$ La nouvelle Ève mère du monde, écrite en janvier 1552.

${ }^{56}$ Les études hébraïques avaient déjà été rétablies en Allemagne par Reuchlin en 1512, en 1517 par Érasme avec la fondation du Collège trilingue de Louvain (latin, grec, hébreu). Postel possédait d'ailleurs un exemplaire du De accentibus et orthographia liguae hebraicae de Reuchlin, paru en 1518 (cf. F. Secret, Bibliographie des manuscrits de Guillaume Postel, Genève, Droz, 1970, p. 60). Sur les textes ésotériques juifs, voir

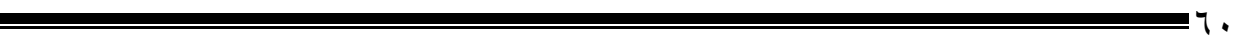


G. G. Scholem, Les origines de la kabbale, Paris, 1966 ou encore La Kabbale et sa symbolique, Paris, Petite Bibliothèque Payot, 1975.

${ }^{57}$ L'Islam était alors quasiment inconnu de la masse des chrétiens et un synode byzantin avait déclaré que le dieu de Mahomet n'était pas le même que celui du christianisme.

${ }^{58}$ Le titre exact en est : De Etruriae regionis, quae prima in orbe europaeo habitata est, originibus, institutis, religione, et moribus, et in primis de aurei saeculi doctrina et uita praestantissima, quae in diuinationis sacrae posita est, commentatio. Cet ouvrage, publié par Lorenzo Torrentino à Florence, a été réédité par G. Cipriani (G. Postel, De Etruriae regionis originibus, institutis, religione et moribus, testo, introduzione, note e commento a cura di Giovanni Cipriani, Roma, C.N.R., (Contributi alla storia degli studi etruschi e italici, 4), 1986).

${ }^{59}$ Édition Cipriani, p. 59-61.

${ }^{60}$ Ibid. p. 199.

«Le dix-septième jour du second mois commença le déluge et Janus, avec toute sa maisonnée, entra dans l'arche préparée pour le salut du genre humain ».

${ }^{61}$ Ibid. p. 134.

Un autre élément d'identification se trouve dans le De originibus... (Paris, D. Lescuyer, 1538), dans lequel Postel rapproche «étymologiquement » Noé de Janus par le fait que le vin, en hébreu, se dit « Jaïn ». Dans son De originibus... (ouvrage comportant un titrerésumé dont la traduction est: «Des origines, ou de l'ancienneté de la langue hébraïque, et de l'affinité des différentes langues; où l'on verra, par l'argument du vocabulaire hébreu, que les colonies ont été conduites du peuple hébreu ou chaldéen dans le monde entier, et par le témoignage des auteurs de l'humanité : on apprendra que les lettres, les lois, et toutes les disciplines en sont redevables ; et qu'il y a une certaine communauté des idiomes méridionaux avec l'hébreu. ») Postel rattache à l'hébreu l'arabe, l'indien, le latin, le français et le grec, mais ne mentionne pas l'étrusque. Sur la démarche bien peu scientifique de l'étymologie postélienne qui préside à cet ouvrage, voir M.-L. Launay, « Le De originibus de 1538 : une rhétorique des origines », Actes du colloque d'Avranches, Paris, Éditions de la Maisnie-Trédaniel, 1985, p. 307-316.

${ }^{62}$ Ibid., p. 131. Sur l'existence de la dodécaple étrusque et sur le nombre et la liste des cités qui peuvent y être rattachées, on consultera par exemple M. Torelli, Storia degli Etruschi, Rome-Bari, Laterza, 1981, qui admet que la fédération d'Étrurie intérieure pouvait regrouper primitivement, c'est-à-dire vers les VII ${ }^{\text {ème }}-\mathrm{VI}^{\mathrm{ème}}$ siècles, Véies, Caeré, Tarquinia, Vulci, Volsinies, Chiusi, Vetulonia, Volterra, Pérouse, Cortone, Arezzo, Fiésole. Cette ligue, unie autour du fanum Voltumnae, a peut-être compté plus de douze cités, tout d'abord parce que certaines villes étrusques, telles Pise, Populonia ou Roselle, paraissent n'avoir acquis leur autonomie par rapport à d'autres cités de l'intérieur que secondairement; ensuite, l'on sait que ce nombre a varié dans le temps: la réorganisation de la ligue des duodecim populi Etruriae sous l'empire romain fait passer le nombre à quinze peuples (avec le titre de praetor Etruriae XV populorum; cf. B. Liou, Praetor Etruriae XV populorum, Bruxelles, 1969) ; enfin, le nombre de douze paraît trop étroitement calqué sur l'organisation fédérale que connaissaient, dans le monde grec, les cités d'Ionie, pour n'être pas quelque peu suspect (indépendamment de son éventuelle valeur religieuse).

63 voir supra note 28 


\section{Traduire l'étrusque : problématiques antiques et modernes}

${ }^{64}$ Cette vision des choses est d'autant plus intéressante - et scientifiquement paradoxale que les Romains de l'Empire, eux, faisaient de la religion étrusque un rempart du paganisme contre le christianisme conquérant; sur la question, voir D. Briquel, Chrétiens et haruspices, Paris, PENS, 1997.

${ }^{65}$ Ibid., p. 239-240 :

"Hinc Christus ipse quum venit Romam iterum in Petro crucifigi elegit ut inversa cruce funderet animam super sacrosantarum Noachi reliquiarum monumentum. 》

${ }^{66} \mathrm{G}$. Cipriani, Il mito etrusco nel rinascimento fiorentino, Florence, Leo S. Olschki, 1980, et C. Vasoli, art. cité supra $\mathrm{n} .49$.

${ }^{67}$ Art. cit. p. $338 s q$.

${ }^{68}$ Sur ce point, nous nous permettons de renvoyer à notre article «La redécouverte des Étrusques par Guillaume Postel », Tôzai, 8, 2006, p. 11-23, en particulier pour sa théorie de la Restitution et la conception d'une monarchie unique qui devait être guidée par la quête de cette Restitution.

${ }^{69}$ V. Georgiev, La lingua e l'origine degli Etruschi, Roma, Nagard, 1979. Les vues de cet auteur paraissent insoutenables, même s'il faut bien reconnaitre que certains mots étrusques offrent des rapprochements intéressants. Ainsi le nom propre de Tarchun (Tarquin) fait-il penser au nom du dieu hittite Tarhundas. Mais l'existence du verbe grec $\tau \alpha \rho \chi \vee \omega$ «rendre les derniers devoirs », qui n'a pas de racine indo-européenne, incite plutôt à chercher une explication par le concept de «péri-indo-européen ». Sur cette notion, voir G. Devoto, Origini indoeuropee, Florence, 1962, p. 116 sq. La piste hittite avait déjà été explorée par A. J. Pfiffig, « Altetruskisch, ein späthethitischer Dialekt ? », Glotta 1965, XLIII, p. 324-332. 